\title{
Produccion En Banano Cavendish Con Desmane Falsa Mas Dos Y Falsa Mas Tres
}

\author{
Ing. Agr. Yary Ruiz Parrales \\ Ing. Agr. Ricardo X. Chávez Betancourt \\ Ing. Agrp. Víctor Molina Barbotó \\ Ing. Agr. Cristina Maldonado Camposano \\ Ing. Agr. David Mayorga Arias \\ Universidad Técnica de Babahoyo (Los Ríos-Ecuador)
}

doi: 10.19044/esj.2016.v12n24p267 URL:http://dx.doi.org/10.19044/esj.2016.v12n24p267

\begin{abstract}
Producers have given importance only to the management of the plantation; however, only a little bit has been done to properly manage the bunch, the same that has affected the quality of the exportable fruit. In this research the trimming of bunches of Cavendish clone was made; the treatments were: False plus three, false plus two and witness (without trimming). The aim in this research was to: Determine whether the practice of hand pruning improves the presentation of the bunch, makes greater fruit and to obtain greater bunch / conversion box. Assess the economic effect of the treatments studied. Once this investigation was done, it was found that the bunch / box conversion increased, and the trimming managed to increase the length of the fingers on the lower hand, increased the weight hand, increased grade and were able to reduce the rejection rate and decline fruit. The results showed statistical differences for the length of fingers and grade, number of hands and weight of hands; however, it showed that it was no significant differences for weight on bunches and rachis. It is important to note that there is greater bunch / conversion box reaching 1.2 and 1.3 for False plus two and False plus three, respectively, while the witness was 0.9; this difference was due to rejection and decline fruit Because it was higher in the witness who did not reach the length or the degree required for export.
\end{abstract}

Keywords: Box, bunch, treatments, weight, export

\section{Resumen}

Los productores han dado importancia solo al manejo de la plantación; sin embargo, poco se ha hecho para manejar adecuadamente del racimo, el mismo que ha afectado la calidad de la fruta exportable. En esta 
investigación realizó el desmane de los racimos del clon Cavendish; los tratamientos son: Falsa más tres, Falsa más dos y testigo (sin desmane), teniendo como objetivo: Determinar si la práctica de poda de manos mejora la presentación del racimo y se consigue mayor grado de los frutos y obtener mayor conversión racimo/caja. Valorar el efecto económico de los tratamientos estudiados Una vez realizada la presente investigación, se observó que se incrementó la conversión racimo/caja, y el desmane logró aumentar la longitud de dedos de las manos inferiores, peso de manos, incremento de grado, se logró disminuir el porcentaje de rechazo y merma. Los resultados indicaron diferencias estadísticas para la longitud de dedos y grado, número de manos y peso de manos; en cambio, no alcanzó diferencias significativas para peso de racimos y raquis. Es importante señalar que hay mayor conversión racimo/caja alcanzando a 1,2 y 1,3 para Falsa más dos y Falsa más tres, respectivamente, mientras el testigo fue 0,9; esta diferencia se debió a que el rechazo y la merma fueron mayores en el testigo que no alcanzaron la longitud, ni el grado exigible para su exportación.

Palabras clave: Caja, racima, tratamientos, peso, exportación

\section{Introduccion}

Los productores del cultivo de banano han dado importancia solo al manejo de la plantación. Sin embargo, poco se ha hecho para manejar adecuadamente el racimo, esto ha afectado la calidad de la fruta exportable y cada vez más se incrementa la demanda del consumo de esta fruta por los países consumidores.

La evolución del mercado internacional de la fruta de banano guarda estrecha relación con la verticalidad de esta industria y su carácter netamente exportador, que depende a su vez de la producción (Martinez et al., 2007).

La cosecha del racimo de banano para exportación se realiza considerando la edad fisiológica del mismo así como el grosor del fruto central de la fila externa de la segunda mano basal, ambos preestablecidos y determinados fundamentalmente en función de la época del año y las especificaciones de los frutos de acuerdo con el mercado de destino (Rica et al., 2014)

Como consecuencia de las exigencias de los mercados de exportación con relación a la longitud mínima de dedos y a un diámetro mínimo, los técnicos bananeros y cultivadores idearon la poda de las manos interiores cuyos dedos en forma general no alcanzan la longitud ni el grado deseado (Salazar \& Arrieta, 2010)

El desmane es una práctica cultural que consiste en eliminar o podar una o varias manos durante la labor de protección de fruta. Se eliminan las manos apicales que generalmente no cumplen con las especificaciones del 
largo del dedo exigidas en los mercados para banano de exportación (Salazar \& Arrieta, 2010).

En esta investigación se tratara de realizar el desmane de los racimos del clon Cavendish; con los tratamientos Falsa más dos, Falsa más tres y testigo (sin desmane).

El trabajo de investigación se precisó entre otros objetivos si la práctica de poda de manos que se utilizaron los racimos del clon cavendish, en los tratamientos de Falsa más dos y Falsa más tres.

Mejora la presentación del racimo y se consigue mayor grado de los frutos, pesos de las manos, longitud de los dedos en las manos en estudio, número de manos, peso de racimos, peso de raquis y obtener mayor conversión racimo/caja y con estos resultados incrementar la producción bananera.

\section{Materiales Y Metodos}

El presente ensayo de investigación se realizó entre los meses de Julio y septiembre de 2011, en los terrenos de la hacienda (La Julia) específicamente en el sector 1, de la Corporación NOBOA S.A., la misma que se encuentra ubicada en el Km 13 de la vía Babahoyo-Quevedo ubicada en Los Ríos-Ecuador.

Este lugar donde se realizó el trabajo de investigación se encuentra a la altura de $7 \mathrm{msnm}$ una temperatura media anual de $26^{\circ} \mathrm{C}$, y una humedad relativa de 86,5\%. Existen dos épocas muy definidas de Diciembre a Mayo (época lluviosa) y de Junio a Noviembre (época seca) y con una precipitación promedio anual de 1262,8 mm situación históricamente normal en esta región.

El terreno donde se realizó este ensayo de investigación, cuenta con todas las obras de infraestructura necesaria que se requieren para la producción tecnificada del cultivo de banano; como también topografía plana, programas de fertilización, sistema de riego sub-foliar, departamento fitosanitario para el control de sigatoka, sistema de drenaje adecuado y cables guía para el transporte de la fruta.

Se seleccionó una plantación de banano establecida con la variedad Cavendish, la misma que presentó una adecuada uniformidad en cuanto a la densidad de siembra y manejo agronómico, los factores en estudio fueron sobre el comportamiento del racimo de banano a la poda de manos inferiores con tres tratamientos: Falsa más dos; Falsa más tres y testigos (sin podar).

Durante la investigación se utilizó la prueba de t de Student apareada, las variables evaluadas fueron las siguientes: Altura de planta a la parición del hijo y a la cosecha (m), Número de hojas funcionales a la parición y a la cosecha, Número de manos por racimo, Longitud de dedos de la segunda y última mano del racimo (cm), Grado de la segunda y última mano, Peso del 
racimo (kg), Número de clúster por plato y Ratio (cajas/racimo). Robert+Wilian Evaluación agronómica y comparativa entre dos variedades de banano (Musa paradisiaca AAA) Aplicando Falsa más dos, Falsa más 3 Tratamientos

\begin{tabular}{|c|c|}
\hline PODA DE MANOS & TRATAMIENTOS \\
\hline 1. Falsa más dos & Poda de tres manos al racimo \\
\hline 2. Falsa más tres & Poda de cuatro manos al racimo \\
\hline 3. Testigo & Racimo sin podar \\
\hline
\end{tabular}

Para este trabajo de investigación se utilizó el diseño experimental "Bloque al azar". En cada tratamiento fue conformado por tres repeticiones y a su vez cada tratamiento por 10 plantas, el área de la parcela experimental fue de 5,2 hectáreas, las mismas que fueron distribuidas de la siguiente manera.

El tratamiento de Falsa más dos se llevó a cabo en un lote de 2,2 hectáreas que corresponde al cable número 20 en el cual también está incluido su testigo. De la misma manera el tratamiento de Falsa más tres se ubicó en el cable continuo es decir el cable 21, en donde también se incluyó el tratamiento testigo alcanzando una superficie de 3 hectáreas utilizando para separar los tratamientos la ayuda de un canal de drenaje tipo secundario que ya estaba establecido en el cultivo.

Fue necesario determinar el color de la cinta en el momento del enfunde para de esta manera identificar los racimos que fueron evaluados hasta el momento de la cosecha, la misma que está organizada por edad, para este trabajo de investigación después se realizó el desmane en racimos de dos semanas de edad es decir, que todas sus manos estén en forma horizontal ya que es la edad adecuada para proceder a realizar la poda de manos inferiores de acuerdo a los tratamientos que fueron establecidos.

Para el caso de Falsa más dos, se eliminó la mano falsa y luego las dos manos contiguas, pero previamente dejando un dedo lateral en la primera mano. Para el caso de Falsa más tres se podó en forma manual la mano falsa y las tres manos contiguas dejando después de la mano falsa los dedos laterales de las dos primeras manos podadas.

Una vez realizada la poda de manos se procedió a identificar a los racimos según el tratamiento, mediante tarjetas que se sujetaron en el raquis junto a la última mano del racimo el tratamiento de Falsa más dos se colocó una tarjeta de color rosado y con otra tarjeta de color amarillo se identificó el tratamiento de Falsa más tres, mientras que el testigo se le colocó una tarjeta de color blanco.

Lo siguiente que se realizó fue utilizar un calibrador tipo pie de rey provisto de Nonio Rectilíneo que permitió realizar calibraciones a diferentes edades de los dedos de las manos que se estudiaron y no se utilizaron los 
calibradores tradicionales ya que estos solo dan calibraciones para cosecha, se evaluaron las dos primeras y dos últimas manos del racimo de banano durante todas las semanas que duró el desarrollo del racimo desde la flor hasta la cosecha.

Una vez cosechados los racimos, estos fueron transportados a la empacadora para su proceso de empaque y en este lugar se efectuó la toma de datos que se investigó entre los parámetros a evaluar fueron: calibración, longitud de dedos, peso de manos, número de manos, peso de racimo, peso de raquis y conversión racimo/caja.

Los datos de cosecha fueron sometidos a análisis de varianza estadística y se utilizó la prueba de Duncan $(\mathrm{P}<0.05)$ para la comparación de significancia estadística al igual que sus respectivos coeficientes de variación.

\section{Resultados}

A continuación se presentan los resultados obtenidos en el presente trabajo de investigación, donde se eliminaron las manos inferiores a los racimos de banano de la variedad Cavendish, los datos evaluados corresponde a: calibración, longitud de dedos, peso de manos, racimos y raquis. Los mismos que fueron sometidos al análisis de la varianza y a la prueba de Duncan.

Para la calibración o grado a la cosecha estos datos fueron sometidos al análisis de la variancia y mostraron diferencia significativas al 95\% de probabilidades. Para la primera mano de acuerdo a la prueba de Duncan el tratamiento Falsa más tres con grado 46 alcanzó el promedio más alto y significativamente diferente solo al testigo que fue de 44. Para la segunda mano con la prueba de Duncan mostró la misma tendencia que en el caso anterior el tratamiento Falsa más tres obtuvo el promedio más alto con grado 46, el mismo que fue significativamente diferente solo para el grado 43 alcanzado por el testigo. En la penúltima mano según Duncan el promedio más alto y significativamente diferente a los demás correspondió al tratamiento Falsa más tres con una calibración de 43; en cambio, el promedio de 41 grados, correspondiente diferente solo al tratamiento testigo en el que sus frutos obtuvo un promedio de 38 grados. Y para el caso de la última mano que también mostró significancia estadística, los promedios correspondientes a los tratamientos Falsa más dos y Falsa más tres que fueron estadísticamente iguales entre si y superior al testigo con grado 38. 
CUADRO 1

Promedio de calibración o grado del fruto de banano a la cosecha obtenida en racimos que fueron sometidos a diferentes tratamientos de desmane.

\begin{tabular}{|c|c|c|c|c|}
\hline \multirow[t]{2}{*}{ TRATAMIENTOS } & \multicolumn{4}{|c|}{ MANOS } \\
\hline & $\begin{array}{c}\text { Pri } \\
\text { me } \\
\text { ra }\end{array}$ & $\begin{array}{c}\text { Seg } \\
\text { und } \\
\text { a }\end{array}$ & $\begin{array}{l}\text { Pen } \\
\text { últi } \\
\text { ma }\end{array}$ & $\begin{array}{c}\text { Ult } \\
\text { im } \\
\text { a }\end{array}$ \\
\hline & 45 & 45 & & 41 \\
\hline Falsa +2 & $\begin{array}{l}\mathrm{ab} \\
46\end{array}$ & $a b$ & $41 \mathrm{~b}$ & $\begin{array}{c}\mathrm{a} \\
42\end{array}$ \\
\hline Falsa +3 & $\begin{array}{c}\mathrm{a} \\
44\end{array}$ & $\begin{array}{c}46 \mathrm{a} \\
43\end{array}$ & $43 \mathrm{a}$ & $\begin{array}{c}\mathrm{a} \\
38\end{array}$ \\
\hline Testigo & bc & bc & $38 \mathrm{c}$ & $\mathrm{b}$ \\
\hline Promedios & 45 & $\begin{array}{c}44, \\
6\end{array}$ & 40,6 & $\begin{array}{c}40 \\
3\end{array}$ \\
\hline & 0,0 & 0,0 & & 0,0 \\
\hline Sig. Est. & 5 & 5 & 0,05 & 5 \\
\hline & 0,7 & 0,7 & & 1,1 \\
\hline CV/\% & 8 & 4 & 1,61 & 4 \\
\hline
\end{tabular}

Posición de mano en el racimo, la primera y segunda son de la parte superior, mientras que la Penúltima y última mano están localizada en la parte inferior del racimo.

Para el análisis de longitud de dedos a la cosecha los datos no alcanzaron diferencias significativas en los tratamientos tal como se aprecia en el cuadro 2.

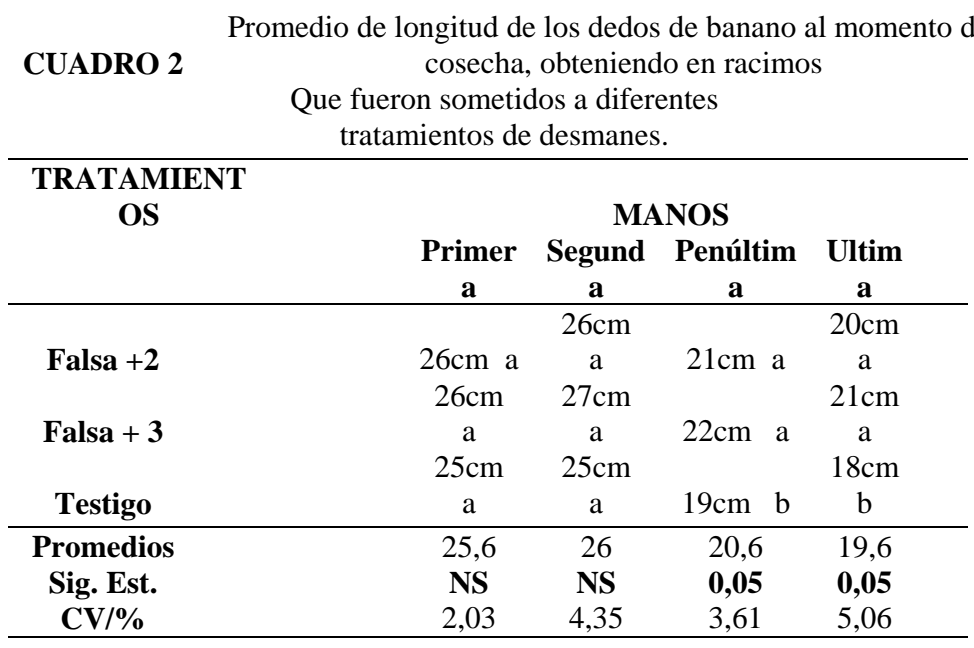

El peso promedio de las manos cosechadas en racimos sometidos a diferentes tratamientos de desmanes mostraron que para la primera mano, los valores al ser analizados estadísticamente mostraron significancia a nivel del 0,05 sus promedios variaron de $5,18 \mathrm{Kg}$ a $5,72 \mathrm{Kg}$ para los tratamientos 
testigos y Falsa más tres. Para el peso de la segunda mano mostró significancia al 95\% de probabilidades y según la prueba de Duncan, los promedios correspondientes a los tratamientos Falsa más dos y Falsa más tres fueron estadísticamente iguales entre sí y diferentes al testigo. Para las ultimas manos, el análisis estadístico mostró diferencias significativas al nivel del 0,05 para la penúltima mano y de 0,01 para la última mano. Cuadro 3

CUADRO 3 Promedios de peso(Kg) de manos al momento de la cosecha de los racimos de banano sometidos A diferentes tratamientos de desmane.

\begin{tabular}{|c|c|c|c|c|}
\hline \multirow[t]{2}{*}{ TRATAMIENTOS } & \multicolumn{4}{|c|}{ MANOS } \\
\hline & Primera & Segunda & Penúltima & Ultima \\
\hline Falsa +2 & $5,18 \quad$ а & $5,0 \quad \mathrm{a}$ & $2,27 \quad b$ & $2,09 \quad b$ \\
\hline Falsa +3 & 5,72 а & 5,45 а & $2,72 \quad a$ & 2,45 a \\
\hline Testigo & 4,48 a & $4,09 \mathrm{~b}$ & $1,81 \mathrm{c}$ & $1,36 \mathrm{c}$ \\
\hline Promedios & 5,22 & 4,81 & 2,27 & 1,95 \\
\hline Sig. Est. & NS & 0,05 & 0,05 & 0,01 \\
\hline CV/\% & 8,9 & 7,9 & 7,9 & 2,29 \\
\hline
\end{tabular}

A lo que respecta al número de manos por racimos, peso de racimo y raquis. Los datos una vez realizado el análisis de la varianza alcanzó diferencias significativas al 95\% de probabilidades solo para el número de manos. Para el peso del racimo se observó una clara tendencia a aumentar el peso de los racimos en los tratamientos que incluían desmane, no así en el tratamiento testigo que se tuvo el menor peso con $37 \mathrm{Kg}$. El coeficiente de variación fue de 5,07\%. Para el peso de raquis los promedios variaron en los tratamientos y testigo y su coeficiente de variación fue de 10,14\% cuadro 4.

CUADRO 4 Promedios de peso(Kg) de racimos y raquis, numero de manos sometidos a diferentes tratamiento De desmanes.

\begin{tabular}{cccccc}
\hline TRATAMIENTOS & Ratio & Número de manos & Peso de Raquis (Kg) & Peso de racimos (Kg) \\
\hline Falsa +2 & 1,2 & 10 b & 4 & 39 \\
Falsa + & 1,3 & $9 \mathrm{~b}$ & 4 & 40 \\
Testigo & 0,9 & $13 \mathrm{a}$ & 3,3 & 37 \\
\hline Promedios & & 10,6 & 3,7 & 38,6 \\
Sig. Est. & & $\mathbf{0 , 0 5}$ & NS & NS \\
CV/\% & & 6,35 & 10,14 & 5,07
\end{tabular}

En el cuadro 5 se muestra el análisis económico obtenido en el ensayo de poda del racimo de banano donde se marca una clara tendencia de conversión racimo/caja y que a pesar de que el testigo mostró mayor número de manos estos no reúnen condiciones para exportación y esto se reflejó que los tratamientos de poda de mano al racimo genera mayor ingreso económico que el testigo. 
CUADRO 5 Análisis económico de rendimiento de banano en el ensayo sometido a diferentes tratamientos de Desmanes.

\begin{tabular}{cccccccc}
\hline TRATAMIENTOS & $\begin{array}{c}\text { Peso De } \\
\text { Racimo }\end{array}$ & $\begin{array}{c}\text { Conversión } \\
\text { Ratio }\end{array}$ & $\begin{array}{c}\text { Producción } \\
\text { Caja/Ha/Año }\end{array}$ & $\begin{array}{c}\text { Incremento } \\
\text { Producción }\end{array}$ & $\begin{array}{c}\text { Costo De La } \\
\text { Labor }\end{array}$ & $\begin{array}{c}\text { Ingreso Total } \\
\text { Ha./Año }\end{array}$ & $\begin{array}{c}\text { Ganancia Neta } \\
\text { Ha./ Año }\end{array}$ \\
\hline Falsa +2 & 39 & 1,2 & 1764 & 441 & 176.800 & $36^{\prime} 514.800$ & $99^{\prime} 128.700$ \\
Falsa + 3 & 40 & 1,3 & 1911 & 588 & 220.500 & $39^{\prime} 557.700$ & $12^{\prime} 171.600$ \\
Testigo & 37 & 0,9 & 1323 & 0 & 0 & $27^{\prime} 386.100$ & 0 \\
\hline
\end{tabular}

Para los cálculos se consideraron 1470 plantas con racimos por año y el valor de la caja 22XU de 43 Lbs. A s/. 20.700 RATIO $=\mathrm{N}^{\circ}$ de cajas exportables $/ \mathrm{N}^{\circ}$ de racimos procesados.

\section{Discusión}

El manejo de racimo de banano, variedad Cavendish, originó diferencias significativas en el grado o calibración de los frutos, hecho que probablemente se basa a la eliminación de una o varias manos del racimo en edades tempranas, su crecimiento y almacenamiento de reservas se incrementa cuando se tiene menor número de manos, esta práctica de desmane temprano acelera el lapso flor-cosecha de 2 a 4 días

También se observaron diferencias significativas en la longitud de los dedos de las dos últimas manos del racimo lo que pone en manifiesto que el desmane permite mayor libertad para que los dedos crezcan y muestren mayor longitud, mientras que cuando no se practica el desmane, estos se presentan con mayor curvatura por lo que no son aceptados por exportación. Estos resultados concuerdan con lo manifestado por Barzola (2).

Cuando en los racimos se eliminaron falsa más dos y falsa más tres en las últimas manos evaluadas mostraron mayor calibración como consecuencia de la acumulación de reservas en los frutos de los racimos desmanados, no así en aquellos que se dejaron todas las manos, estas mostraron menor peso; es decir en este caso las reservas se traslocan para mantener un mayor número de frutos siendo estos de mayor tamaño y las últimas manos del racimo no alcanzaron la calibración necesaria para ser calificadas de exportación.

El número de manos, en cambio como era de esperarse fue mayor en el tratamiento testigo en que no se practicó desmane alguno; en este caso, el mayor número de manos no significó que todos son exportables por no presentar la calidad son eliminados o rechazados por no presentar calidad exigida para que sean considerados de exportación.

El peso del racimo no mostró significancia estadística entre los tratamientos; sin embargo, se obtuvo mayor peso de frutos con falsa más dos y falsa más tres con 39 y $40 \mathrm{Kg} / \mathrm{racimo}$, mientras que el testigo fue de $37 \mathrm{Kg}$. La diferencia aparentemente pequeña en el peso de los racimos, se puede explicar que no concuerdan con el número de manos de este tratamiento puesto que para el testigo que pesa de $37 \mathrm{Kg}$ de fruta corresponden 13 
manos, mientras que en Falsa más dos y Falsa más tres 39 y 40 Kg de fruta, corresponden a 10 y 9 manos respectivamente.

En el caso del testigo se mantiene en un ratio de 0,9 en tanto que con el desmane el ratio se registra sobre 1,2 a 1,3 lo que significa que sus frutos son de mayor calidad tanto por longitud, como por grado. Resultados similares fueron reportados por Barzola (2).

\section{Conclusion}

En base a los resultados obtenidos se puede señalar las conclusiones siguientes:

- $\quad$ La eliminación temprana de Falsa más dos y Falsa más tres en racimo de banano originó mayor grado o calibración de la fruta. También se observó que aceleró el lazo flor-cosecha en 2 y 4 días mediante desmane.

- La mayor longitud de los dedos se alcanzó cuando se practicó el desmane al racimo; en cambio el tratamiento testigo en el que no se eliminaron manos se observó que sus manos presentaron dedos más curvos.

- $\quad$ El peso de manos fue mayor con el desmane temprano del racimo.

- $\quad$ No se encontraron diferencias significativas para peso de racimo y/o raquis.

- En cuanto al número de manos se obtuvieron diferencias significativas, mostrando mayor número de estas en el tratamiento testigo, sin desmanes.

- $\quad$ El ratio fue de 0,9 para el tratamiento testigo y de 1,2 a 1,3 para los tratamientos que se eliminaron 3 y 4 manos/racimos.

\section{Recomendaciones}

- Incluir otras prácticas de manejo del racimo además del desmane.

- Validar estos datos otras condiciones de clima y suelo.

- $\quad$ Estudiar el efecto del manejo del racimo utilizando Falsa más tres en plantas que se realizan resiembra o renovación parcial en la plantación.

- Difundir estos resultados en beneficio de los productores de banano.

\section{References:}

Barrera, J. L., Salazar, C. F., \& Arrieta, K. L. (2010). Efecto del desmane yremoción de dedos sobre la calidad y producción del banano. Temas agrarios, 15(2), 58-65.

Haddad, O., Surga, J., González, M., Del Valle, M., Pargas, R., Manzanilla, E., \& Rodríguez, H. (1991). Comportamiento del banano (Giant cavendish) bajo las condiciones de los valles de Aragua con fines de exportación.[Performance of bananas (Giant Cavendish) grown in Aragua 
valleys for export]. Jornadas técnicas CENIAP Maracay (Venezuela). May 1991.

Hernández, C. (2011). Evaluación de diferentes tipos de podas en glomérulos y frutos laterales de los glomérulos de racimos de banano (Musa sp.) y su efecto en la calidad y el aprovechamiento de la fruta en la finca Hopy, “entre Rios”, Puerto Barrios, Izabal, Guatemala, C.A. Tesis Ingeniero Agrónomo. Universidad de San Carlos de Guatemala, Facultad de Agronomía. Instituto de Investigaciones Agronómicas. 11 - 12 pp.

Calvo, A. V. (2010). Efecto del desmane y de la modalidad de cosecha sobre las características y producción de racimos de plátano tipo Francés FHIA-21. SOMMAIRE/INHOUD/SUMARIO, 28(1), 16-23.

Martinez, G., Delgado, E., Pargas, R., Manzanilla, E., Ramírez, H., \& Inia-ceniap, T. A. I. (2007). Consideraciones generales sobre la producción y el comercio mundial de banano . I: Producción, exportación e importación, 1-11.

INTA, (2013). Instituto Nacional de Tecnología Agropecuaria, Argentina. Cultivo de banano. Disponible en: http://inta.gob.ar/documentos/cultivo-delbanano.

López, M. (2014). Evaluación de Métodos de desmane con aplicación de ácido giberelico en el racimo de banano Musa AAA; Santo Domingo, Suchitepéquez. Tesis Ingeniero.

Rica, U. D. C., Rica, U. D. C., Rica, C., Del, E., Intensivo, D., El, S., ... Banano, R. D. E. (2014). Disponible en: http://www.redalyc.org/articulo.oa?id=43730495009.

Salazar, C. F., \& Arrieta, K. L. (2010). Calidad Y Producción Del Banano Effect Of Dehanding And Removal Of Fingers On The Quality And Production Of Banana Tree. 